\title{
Predicción de la accidentabilidad en función de los comportamientos arriesgados y agresivos al volante: Diferencias según la edad y el género
}

\author{
David Herrero-Fernández, Mireia Oliva-Macías y Pamela Parada-Fernández
}

Universidad Europea del Atlántico, Santander, España

\begin{abstract}
Prediction of accident rate from risky and aggressive behavior behind the wheel: Differences by age and gender. Road accidents are one of the main causes of death in Spain. Risky and aggressive behavior behind the wheel are two of the most relevant predictive variables. This study had three aims: First, the psychometric adaptation of the Driving Survey (DS) in a Spanish sample; second, the analysis of the differences by age and gender in risky and aggressive driving, as well as in the self-perception of the driver and in the accident rate; and third, the analysis of the prediction of the accident rate based on the other variables. The results showed a factorial distribution, similar to the original version, with adequate values of both internal consistency and validity. Men scored higher than women in risky driving, while the youngest drivers scored higher in almost all variables. Finally, it was observed that both risky and aggressive behavior predicted accident rate significantly.
\end{abstract}

Keywords: Risky driving; aggressive driving; accident rate; driving survey.

Resumen: Los accidentes de tráfico constituyen una de las principales causas de muerte en España. Los comportamientos arriesgados y agresivos al volante se han perfilado como dos de las variables más relevantes. La presente investigación tuvo tres objetivos. Primero, la adaptación psicométrica del Driving Survey (DS) en una muestra española; segundo, el análisis de las diferencias por edad y género en comportamientos arriesgados y agresivos al volante, así como en la accidentabilidad; y tercero, el análisis de la predicción de la accidentabilidad en base a las anteriores variables. Los resultados mostraron una distribución factorial similar a la versión original, con unas propiedades psicométricas adecuadas en términos de consistencia interna y de validez. Los hombres puntuaron más alto en aspectos relacionados con conducción arriesgada, mientras que los más jóvenes puntuaron más alto en casi todos los indicadores evaluados. Finalmente, se encontró que tanto los comportamientos arriesgados como los agresivos predecían significativamente la accidentabilidad.

Palabras clave: Conducción arriesgada; conducción agresiva; accidentabilidad; encuesta de conducción.

\section{Introducción}

Los accidentes de tráfico constituyen una de las principales causas de muerte en el mundo. En España, a pesar de que el número de fallecidos desciende año tras año (p.ej., en 2017 se contabilizaron 1830 personas, siendo un $3.84 \%$ menos que en 2012 , cuando se contabi-

Recibido: 07 de enero 2019: aceptado: 09 de mayo 2019

Correspondencia: David Herrero-Fernández, Facultad de Ciencias de la Salud, Universidad Europea del Atlántico, C/ Isabel Torres 21, 37011 Santander, España. Correo-e: david.herrero@uneatlantico.es lizaron 1903), el número de accidentes de tráfico no sólo no varía, sino que incluso en ocasiones las cifras revelan incrementos con respecto a años anteriores. En este caso, en 2017 se produjeron 102.233 accidentes, siendo un $18.71 \%$ más que el año 2012, cuando se contabilizaron 83.115 (DGT, 2017).

El estudio de la accidentabilidad se ha fundamentado en el análisis de los tres pilares que conforman la seguridad vial: el factor vehículo, el factor vía y el factor humano, que engloba los aspectos psicológicos del usuario, en este caso el conductor (Herrero-Fernández, 2017). De los tres, el factor humano es el más relevante a la 
hora de pronosticar los accidentes de tráfico (Brewer, 2000). Dentro del factor humano se han destacado variables como el estrés, la fatiga y las distracciones, que se han asociado con el decremento en la conducción segura y con una mayor propensión a comportamientos arriesgados y agresivos (Backaitis, 2006; Montoro, Useche, Alonso, y Cendales, 2018; Smith, 2016). Son precisamente las conductas arriesgadas y agresivas las que más intensamente se han vinculado con la accidentabilidad. Específicamente, se han llevado a cabo estudios que han hallado la relación entre la probabilidad de sufrir un accidente y variables tales como la intencionalidad de cometer violaciones contra el código de circulación (Elander, West, y French, 1993), factores de personalidad como el rasgo de ira general y el rasgo de ira en la conducción (Deffenbacher, Lynch, Filetti, Dahlen, y Oetting, 2003), y la impulsividad y la búsqueda de sensaciones (Schwebel et al., 2007). Por otro lado, a pesar de la aparente similitud entre los comportamientos arriesgados y agresivos, es importante diferenciarlos. Mientras que en el caso de los comportamientos arriesgados la motivación fundamental de quien los comete es la búsqueda de sensaciones, sin existir intencionalidad de herir, ofender o molestar a un tercero, en el caso de los comportamientos agresivos la principal característica es precisamente esa intencionalidad de causar un daño físico o psicológico al objeto de los mismos (Baron y Richardson, 1994; Berkowitz, 1993). Por tanto, un mismo comportamiento podría ser arriesgado o agresivo, en función de la motivación de quien lo comete. Incluso, además de la posible similitud conductual de ambos tipos de comportamiento, se ha observado la estrecha relación entre riesgo y agresión al volante, de modo que los conductores más propensos a comportarse arriesgadamente lo serán también a hacerlo agresivamente (Herrero-Fernández, 2016; Iliescu y Sârbescu, 2013).

Por lo que respecta a las variables demográficas, cabe destacar los efectos que tienen sobre la conducción arriesgada y agresiva el género y la edad. Con respecto al género, en referencia a los comportamientos agresivos los resultados no son concluyentes. Algunos estudios sugieren que los hombres experimentan niveles más altos de ira y comportan más agresivamente al volante que las mujeres (Vanlaar, Simpson, Mayhew, y Robertson, 2008). Otros, en cambio, han encontrado que son las mujeres las más propensas a experimentar ira (Sullman, Stephens, y Yong, 2014). Finalmente, otros estudios no han encontrado diferencias entre hombres y mujeres, o los tamaños de efecto han sido muy bajos (Herrero-Fernández, 2011a, 2011b). Con respecto a los comportamientos arriesgados, nuevamente las diferencias no están claras. Mientras que en unos estudios se llega a la conclusión de que las mujeres tienden más a distraerse que los hombres (Bone y Mowen, 2006; Schwebel, Severson, Ball, y Rizzo, 2006), en otros se observa que los hombres tienen una mayor habilidad autopercibida y tienden a realizar más conductas de riesgo que las mujeres (Rhodes y Pivik, 2011).

Por lo que respecta a la edad, los resultados son mucho más claros. La gran mayoría de investigaciones han encontrado relaciones inversas entre esta variable y la conducción arriesgada y agresiva, observándose que a mayor edad menor es la frecuencia de dichas conductas (Herrero-Fernández y Fonseca-Baeza, 2017).

Por tanto, teniendo en cuenta la relevancia de los comportamientos arriesgados y agresivos en la predicción de los accidentes de tráfico, surge la necesidad de desarrollar instrumentos de evaluación fiables y válidos que posibiliten el estudio de estos constructos. Con este fin se han diseñado cuestionarios desde distintas perspectivas teóricas. Uno de los más completos, por valorar comportamientos arriesgados y agresivos y situaciones directamente relacionadas con la accidentabilidad, es el Driving Survey (DS) (Deffenbacher, Huff, Lynch, Oetting, y Salvatore, 2000). Este instrumento consta de tres partes. La primera parte incluye 4 ítems independientes en formato de escala visual - analógica que evalúan la auto percepción del conductor en las dimensiones calmado-colérico, poco agresivo-muy agresivo, cauteloso-temerario y seguro-inseguro. La segunda parte incluye 28 ítems que se agrupan en dos factores denominados "comportamientos agresivos" (13 ítems, $\alpha=.85$ ) y "comportamientos arriesgados" (15 ítems, $\alpha=.86$ ). Finalmente, la tercera parte incluye 6 ítems independientes que evalúan situaciones relacionadas con la accidentabilidad, de manera que 3 de ellos preguntan por cuestiones referidas al último mes, y los otros 3 ítems lo hacen en referencia a cuestiones del último año. Según el estudio en el que se propuso este instrumento y en otros posteriores (Deffenbacher, Deffenbacher, Lynch, y Richards, 2003; Deffenbacher, Filetti, Richards, Lynch, y Oetting, 2003; Deffenbacher, Lynch, et al., 2003), estos 6 ítems son independientes por tener una consistencia interna muy baja $(\alpha=.45)$.

Con todo esto, los objetivos del presente estudio son tres. En primer lugar, analizar las propiedades psicométricas (estructura factorial, consistencia interna y validez convergente y divergente) del DS en una muestra española, bajo la hipótesis inicial de que los 28 ítems correspondientes a conductas arriesgadas y agresivas al volante se agruparán o bien en dos factores (de acuerdo con el modelo original) o bien en uno solo (tanto por ser la estructura más parsimoniosa posible, como por la similitud conductual que en ocasiones sucede entre comporta- 
mientos arriesgados y agresivos, tal como se ha expuesto, a pesar de las importantes diferencias en el plano motivacional e intencional). En segundo lugar, analizar las diferencias por edad y género en comportamientos arriesgados y agresivos, y en situaciones relacionadas con la accidentabilidad. En tercer lugar, analizar la predicción de los comportamientos arriesgados y agresivos sobre las situaciones relacionadas con la accidentabilidad.

\section{Método}

\section{Participantes}

La muestra final estuvo formada por 404 participantes, seleccionados según un tipo de muestreo no probabilístico (incidental por conveniencia) de la población general de dos regiones de España: a Comunidad Autónoma de Valencia y la Comunidad Autónoma del País Vasco. Los requisitos para participar en el estudio eran disponer de permiso de conducción $\mathrm{B}$ vigente y conducir al menos una vez por semana. Del total de los participantes, $190(47.03 \%)$ eran hombres y los 214 (52.97\%) restantes mujeres. La edad osciló entre 18 y 70 años $(M=$ $37.59, D T=13.93)$, y los años de experiencia como conductor oscilaron entre 0 y $50(M=17.38, D T=13.23)$. Todos los participantes fueron voluntarios y no recibieron ningún tipo de compensación.

\section{Procedimiento}

En primer lugar, se procedió a adaptar lingüísticamente el Driving Survey a través del procedimiento de traducción-retrotraducción (Hambleton, 1996). Un primer traductor tradujo el original en inglés al castellano, y posteriormente un segundo traductor tradujo esa versión en castellano al inglés. Seguidamente se comparó la versión inglesa original con la versión inglesa resultante del segundo traductor, corrigiendo los matices diferenciales entre ambas versiones con el fin de lograr la mayor equivalencia posible en el significado de los ítems. Ambos traductores eran titulados en Filología Inglesa. Finalmente, los ítems que incluían originalmente unidades de velocidad en millas por hora fueron traducidas por su equivalente en kilómetros por hora, según las pautas establecidas por el Sistema Internacional de Medidas.

Una vez obtenida la versión final del cuestionario en castellano, se procedió a la aplicación. Ésta fue llevada a cabo bien individualmente, bien en grupo, en función de la disponibilidad de los participantes. Este procedimiento se ha seguido en estudios similares (Herrero-Fernández, 2011a), justificándose por la sencillez de la tarea y porque la mayoría de cuestionarios de personalidad puedes en aplicados indistintamente de ambas maneras. Finalmente, al comienzo del protocolo de cuestionarios se incluyó, a modo de consentimiento informado, una hoja informativa con los objetivos generales del estudio y el tipo de cuestiones que se iban a plantear. Igualmente, se informaba de la voluntariedad de la participación y se garantizaba el anonimato y la confidencialidad de los datos.

\section{Instrumentos}

Driving Survey (DS; Deffenbacher et al., 2000; versión en español a desarrollar en el presente estudio según se detalla en el procedimiento). Tal como se ha expuesto, este instrumento consta de 38 ítems. Los 4 primeros (escalas visual - analógicas de 7 puntos, siendo categorías puntuables de 0 a 6) se refieren a la autopercepción del conductor en los continuos calmado-colérico, poco agresivo-muy agresivo, cauteloso-temerario y seguro-inseguro. Los 28 siguientes valoran conductas arriesgadas y agresivas, preguntándose el número de veces que se llevó a cabo cada uno de los comportamientos con 6 opciones de respuesta (entre 0 veces y 5 o más veces). Finalmente, los 6 últimos ítems valoran el número de veces que han ocurrido una serie de eventualidades directamente relacionadas con la accidentabilidad, tres de ellas referidas al último mes, y las otras tres referidas al último año. Al igual que en el anterior caso, existen seis opciones de respuesta (entre 0 veces y 5 o más veces).

Driving Log (DL; Deffenbacher et al., 2000). Se utilizó la versión española del DL (Herrero-Fernández, Fonseca-Baeza, y Pla-Sancho, 2014), realizada a partir de la versión original. Este cuestionario valora los comportamientos arriesgados y agresivos ocurridos durante un día completo. Consta de dos partes: la primera corresponde al número de veces que la persona condujo en el día, la distancia total que recorrió y el número de veces en que experimentó ira. La segunda parte consiste en 19 ítems que se agrupan en dos factores (comportamientos arriesgados, 12 ítems, $\alpha=.72$ con los datos del presente estudio, y comportamientos agresivos, 9 ítems, $\alpha=.73$ igualmente con los datos del presente estudio) El estilo de respuesta es dicotómico, de manera que la persona tiene que indicar si los llevó a cabo o no en el día en cuestión.

\section{Análisis estadístico}

Los análisis estadísticos comprendieron tres partes principales. En la primera parte, se analizaron los aspectos descriptivos de los ítems y los factores esperados, y 
se analizaron las propiedades psicométricas del instrumento. Por parte de los estadísticos descriptivos se calculó la media, desviación típica, asimetría y curtosis. En cuanto al estudio de las propiedades psicométricas del DS, primeramente se calculó la consistencia interna (a de Cronbach) de los factores hipotetizados, y seguidamente se llevó a cabo el análisis factorial confirmatorio (AFC) mediante el software EQS 6.1. Concretamente, se llevaron a cabo dos AFC, comparando dos estructuras. Primero se analizó la estructura bifactorial, análoga a la versión original del instrumento, y seguidamente se analizó el ajuste del modelo más parsimonioso posible, en el que todos los ítems saturarían en un único factor. Una vez elegido el moldeo con mejor ajuste, se calcularon los coeficientes de fiabilidad del constructo (FC) y la varianza media extraída (AVE) a partir de los pesos factoriales y los errores de estimación de los ítems. Teniendo en cuenta que no se cumplió el requisito de la normalidad multivariada, se siguió el método de mínimos cuadrados no ponderados para la estimación de los parámetros, por ser el más recomendado ante el incumplimiento de la normalidad (Bollen, 1989). Por otro lado, se analizaron cuatro índices de bondad de ajuste para valorar las estructuras factoriales. En primer lugar, el cociente entre Satorra-Bentler $\chi^{2}\left(\mathrm{~S}-\mathrm{B} \chi^{2}\right)$ y los grados de libertad, que debe ser menor de 2 para poder aceptar el ajuste del modelo; el índice de ajuste comparativo (CFI) y el índice de ajuste no-normalizado (NNFI), que han de ser superiores a .90 , y el error cuadrático medio de aproximación (RMSEA), que ha de ser inferior a .08.

Seguidamente se procedió a analizar la validez convergente y discriminante del cuestionario. Para ello se calcularon los coeficientes de correlación ( $r$ de Pearson) entre los factores resultantes del DS, los ítems relativos a la accidentabilidad y los ítems relativos a la autopercepción del conductor con los factores del DL (Comportamientos agresivos y Comportamientos arriesgados).

En la segunda parte de los resultados se analizó la influencia de la edad y el género en los comportamientos arriesgados y agresivos según el DS. Para ello se llevó a cabo un MANOVA de 2 (género) $\times 4$ (edad), introduciendo como variables dependientes los dos factores del DS y los ítems relativos a la accidentabilidad y a la autopercepción del conductor en las escalas visual-analógicas. Se calculó tanto la significación como el tamaño del efecto $\left(\eta^{2}\right)$, que fue interpretado según el criterio de Cohen, de modo que valores entre .01 y .04 se consideraron bajos; entre .05 y .14 medios, y mayores de .14 altos (Cohen, 1988).

Finalmente, en la tercera parte se aplicaron modelos de regresión múltiple con el fin de valorar hasta qué punto los comportamientos arriesgados y los comporta- mientos agresivos pronosticaban la accidentabilidad. Teniendo en cuenta que los seis ítems que representan eventos relacionados con la accidentabilidad son considerados independientes, se estableció un total de seis modelos de regresión. Los análisis de regresión se realizaron mediante el método de introducir, siendo las variables independientes la edad, el género, la puntuación obtenida en comportamientos arriesgados y en comportamientos agresivos en el DS, y la puntuación en las cuatro escalas visual-analógicas de dicho instrumento. Como variables dependientes se incluyeron, respectivamente, cada uno de los 6 items que valoran la accidentabilidad.

\section{Resultados}

\section{Estadísticos descriptivos y propiedades psicométricas}

Con respecto a los estadísticos descriptivos, los resultados se indican en la Tabla 1. Tal como se puede observar, las medias de los ítems oscilaron entre 0.05 (ítem 38) y 1.96 (ítem 1). Los coeficientes de asimetría muestran una elevada asimetría positiva $(>1.00)$ en la mayoría de ítems, así como en los dos factores hipotetizados. De igual modo, los coeficientes de curtosis indican un elevado apuntamiento (distribución leptocúrtica). Esto implica que no se puede asumir la distribución normal de las variables.

Seguidamente se llevó a cabo un análisis de la consistencia interna ( $\alpha$ de Cronbach) de los factores hipotetizados, siguiendo la estructura de la versión original (Deffenbacher et al., 2000). De esta forma, se verificó la elevada consistencia interna de los factores Conducción Agresiva (12 ítems, uno suprimido por la baja correlación que mostró con el resto de la escala, $\alpha=.82$ ) y Conducción Arriesgada (15 ítems, $\alpha=.80$ ), a la vez que el tercer factor probado en el estudio original, Accidentabilidad, mostró una consistencia interna muy baja $(\alpha=.46)$. Por este motivo y de acuerdo con la estructura original, los 6 ítems correspondientes a la accidentabilidad fueron considerados independientes.

Posteriormente se llevó a cabo el análisis factorial confirmatorio sobre los 27 ítems, que hipotéticamente se agruparían en dos factores (comportamientos arriesgados y comportamientos agresivos). Tal como cabía esperar en base a los análisis univariados de asimetría y curtosis, el coeficiente de curtosis multivariada mostró el incumplimiento de la normalidad, $\beta_{2, \mathrm{p}}=1192.95$ $p<.001$. De este modo, se comparó el ajuste de dos modelos. El primero analizó la estructura más parsimoniosa posible, de un solo factor. Sin embargo, ninguno de los índices alcanzó los niveles esperables $\left(\mathrm{S}-\mathrm{B} \chi^{2} / \mathrm{gl}=\right.$ 
Tabla 1. Ítems, estadísticos descriptivos y factores hipotetizados del cuestionario Driving Survey

\begin{tabular}{|c|c|c|c|c|}
\hline & $M$ & $D T$ & Asimetría & Curtosis \\
\hline \multicolumn{5}{|l|}{ Como conductor, soy... } \\
\hline 1. Muy calmado - Muy colérico & 1.95 & 1.22 & 0.26 & -0.61 \\
\hline 2. Poco agresivo - Muy agresivo & 1.60 & 1.29 & 0.74 & 0.07 \\
\hline 3. Muy cauteloso - Muy temerario & 1.76 & 1.23 & 0.52 & -0.08 \\
\hline 4. Muy seguro - Muy inseguro & 1.55 & 1.27 & 0.92 & 0.71 \\
\hline \multicolumn{5}{|l|}{ En el último mes, ¿cuántas veces has... } \\
\hline 5. Perdido la concentración mientras conducías? & 1.82 & 1.72 & 0.61 & -0.87 \\
\hline $\begin{array}{l}\text { 6. Tenido una pequeña pérdida de control del vehículo que esta- } \\
\text { bas conduciendo? }\end{array}$ & 0.42 & 0.93 & 2.61 & 6.82 \\
\hline 7. Estado a punto de tener un accidente? & 0.31 & 0.78 & 3.44 & 14.24 \\
\hline \multicolumn{5}{|l|}{ En el último mes, ¿cuántas veces... } \\
\hline 8. Rompiste o dañaste una parte del vehículo?a & 0.11 & 0.46 & 6.33 & 50.76 \\
\hline 9. Tuviste una discusión con un pasajero mientras conducías? & 0.44 & 0.85 & 2.18 & 4.90 \\
\hline 10. Tuviste una discusión con el conductor de otro vehículo? & 0.22 & 0.63 & 3.87 & 19.23 \\
\hline 11. Te peleaste con el conductor de otro vehículo? & 0.07 & 0.35 & 6.95 & 58.65 \\
\hline 12. Hiciste un gesto de enfado a otro conductor o peatón? & 0.62 & 1.15 & 2.33 & 5.69 \\
\hline 13. Insultaste a otro conductor o peatón?? & 0.71 & 1.22 & 2.05 & 3.86 \\
\hline 14. Diste destellos con las luces largas para expresar enfado? & 0.36 & 0.78 & 2.44 & 6.25 \\
\hline 15. Tocaste el claxon con enfado? & 0.52 & 0.93 & 2.37 & 6.61 \\
\hline 16. Gritaste a otro conductor o peatón? & 0.49 & 1.02 & 2.43 & 6.04 \\
\hline 17. Condujiste estando muy cabreado? & 0.53 & 0.97 & 2.15 & 4.97 \\
\hline 18. "Te saliste de tus casillas" de lo enfadado que estabas? & 0.11 & 0.44 & 4.68 & 23.03 \\
\hline 19. Condujiste muy pegado a otro vehículo de lo enfadado que estabas? & 0.12 & 0.47 & 5.25 & 37.17 \\
\hline 20. Cortaste por delante la trayectoria de otro vehículo? & 0.10 & 0.47 & 6.10 & 44.56 \\
\hline \multicolumn{5}{|l|}{ En el último mes, ¿cuántas veces has... } \\
\hline 21. Conducido sin llevar puesto el cinturón de seguridad? & 0.42 & 1.19 & 3.03 & 8.23 \\
\hline 22. Conducido habiendo bebido previamente? & 0.34 & 0.90 & 3.43 & 12.63 \\
\hline 23. Estado bebiendo y conducido? & 0.14 & 0.59 & 5.52 & 34.45 \\
\hline 24. Conducido $15-30 \mathrm{Km} / \mathrm{h}$ por encima de lo permitido? & 1.64 & 1.84 & 0.78 & -0.82 \\
\hline 25. Conducido más de $30 \mathrm{Km} / \mathrm{h}$ por encima de lo permitido? & 0.69 & 1.45 & 2.13 & 3.31 \\
\hline 26. Conducido de forma insegura? & 0.41 & 0.84 & 2.83 & 9.89 \\
\hline 27. Seguido a otro vehículo demasiado cerca? & 0.36 & 0.85 & 3.04 & 10.60 \\
\hline 28. Cambiado de carril de forma insegura? & 0.40 & 0.85 & 2.75 & 8.74 \\
\hline 29. Desviado hacia el carril contiguo? & 0.35 & 0.87 & 3.07 & 10.23 \\
\hline 30. Cambiado de carril par air más rápido habido tráfico lento? & 1.08 & 1.51 & 1.32 & 0.70 \\
\hline 31. Saltado un semáforo en rojo o una señal de STOP? & 0.42 & 0.97 & 3.05 & 10.24 \\
\hline 32. Hecho un giro ilegal? & 0.49 & 0.96 & 2.30 & 5.55 \\
\hline 33. Conducido peligrosamente? & 0.20 & 0.66 & 4.07 & 19.34 \\
\hline 34. No respetado un semáforo o una señal de STOP? & 0.38 & 1.01 & 3.29 & 11.13 \\
\hline 35. Entrado en una intersección habiendo semáforo en rojo? & 0.12 & 0.53 & 5.81 & 39.16 \\
\hline \multicolumn{5}{|l|}{ En el último año, ¿cuántas veces... } \\
\hline 36. Te han multado por cometer una infracción al volante? & 0.11 & 0.46 & 6.16 & 48.76 \\
\hline 37. Has tenido un accidente leve? & 0.28 & 0.63 & 3.28 & 15.30 \\
\hline 38. Has tenido un accidente grave? & 0.05 & 0.30 & 8.54 & 89.61 \\
\hline \multicolumn{5}{|l|}{ Factores hipotetizados } \\
\hline Comportamiento agresivo & 4.29 & 5.77 & 2.17 & 5.88 \\
\hline Comportamiento arriesgado & 7.41 & 8.14 & 1.72 & 3.53 \\
\hline
\end{tabular}

${ }^{a}$ El ítem 8 fue eliminado por su baja correlación con el total del factor. 
3.77; $\mathrm{CFI}=.79 ; \mathrm{NNFI}=.78 ; \mathrm{RMSEA}=.083(90 \% \mathrm{IC}:$ .078 - .088). El segundo de los modelos probados analizó el ajuste en dos factores correlacionados, siguiendo el modelo original (Figura 1). En este caso todos los índices se encontraron dentro de los límites admisibles $\left(\mathrm{S}-\mathrm{B} \chi^{2} / \mathrm{gl}=1.88 ; \mathrm{CFI}=.94 ; \mathrm{NNFI}=.93 ; \mathrm{RMSEA}=\right.$ .047 (90\% IC: .041 - .053). Además, se comprobó que este último modelo ajustaba significativamente mejor que el primero, $\chi^{2}(2)=569.99, p<.001$, de modo que se confirma la estructura bifactorial del cuestionario, considerando independientes los 6 ítems relativos a la accidentabilidad y las 4 escalas visual-analógicas relativas a la autopercepción del conductor. En base a los pesos factoriales y a los errores de estimación se calculó la FC y la AVE. En el caso del factor Comportamiento arriesgado, $\mathrm{FC}=.77, \mathrm{AVE}=.21$, mientras que en el caso de factor Comportamiento agresivo, $\mathrm{FC}=.80 \mathrm{y}$ $\mathrm{AVE}=.26$.

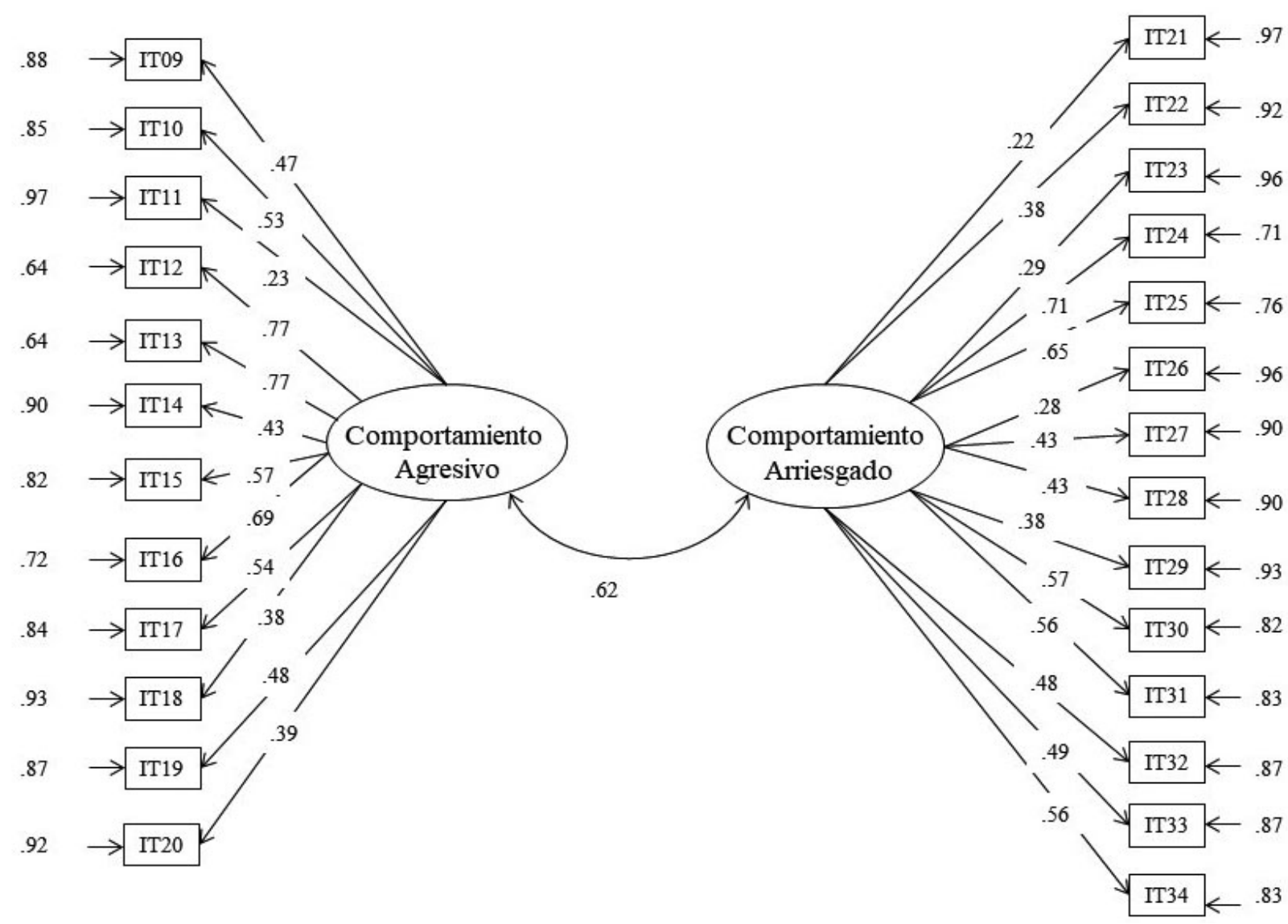

Figura 1. Diagrama de flujos relativo a los ítems y factores del cuestionario Driving Survey. Nota: Se especifican los pesos factoriales (coeficientes lambda estandarizados, siendo todos ellos significativos), los errores de estimación y la correlación entre factores

En cuanto al análisis de validez convergente y discriminante del DS, los resultados se detallan en la Tabla 2. Tal como se puede observar, se evidenció convergencia entre los factores relativos al Comportamiento Arriesgado y al Comportamiento Agresivo del DL y del DS, al ser más fuertes los coeficientes de correlación entre factores análogos que con el resto de las variables. Igualmente, el número de veces que se experimentó ira en el día valorado (DL) correlacionó más fuertemente con el factor Comportamiento agresivo del DS que con el resto de variables. Sin embargo, aunque de forma más débil, se observaron también correlaciones positivas y signifi- cativas entre factores relativos al riesgo y factores relativos a la ira y agresión.

\section{Diferencias por edad y género}

Seguidamente se analizó la influencia de la edad y el género en los comportamientos arriesgados y agresivos según el DS. Los resultados evidenciaron que no existía un efecto de interacción significativo entre los dos factores, $F(36,1143)=1.18, p=.217$. Sin embargo, sí se apreció un efecto significativo tanto por género, $F(12,379)=6.12, p<$ $.001, \eta^{2}=.16$, como por grupos de edad, $F(36,1143)=$ 
Tabla 2. Correlaciones ( $r$ de Pearson) entre el cuestionario Driving Survey (DS) y el cuestionario Driving Log (DL)

\begin{tabular}{|c|c|c|c|c|c|c|c|c|c|c|c|c|c|c|}
\hline & $\begin{array}{c}\text { Agresión } \\
\text { DS }\end{array}$ & $\begin{array}{c}\text { Riesgo } \\
\text { DS }\end{array}$ & DS1 & DS2 & DS3 & DS4 & DS5 & DS6 & DS7 & DS36 & DS37 & DS38 & $\begin{array}{l}\text { Veces } \\
\text { Ira }\end{array}$ & $\begin{array}{c}\text { Riesgo } \\
\text { DL }\end{array}$ \\
\hline Agr. DS & - & & & & & & & & & & & & & \\
\hline Ries. DS & $.57 * * *$ & - & & & & & & & & & & & & \\
\hline DS1 & $.46^{* * *}$ & $.30 * * *$ & - & & & & & & & & & & & \\
\hline DS2 & $.47 * * *$ & $.34 * * *$ & $.73^{* * *}$ & - & & & & & & & & & & \\
\hline DS3 & $.29 * * *$ & $.41 * * *$ & $.44 * * *$ & $.47^{* * * *}$ & - & & & & & & & & & \\
\hline DS4 & .05 & .05 & $.26^{* * *}$ & $.19^{* * *}$ & $.30 * * *$ & - & & & & & & & & \\
\hline DS5 & $.32 * * *$ & $.35^{* * *}$ & $.31^{* * *}$ & $.25^{* * *}$ & $.26^{* * *}$ & .09 & - & & & & & & & \\
\hline DS6 & $.31 * * *$ & $.33 * * *$ & $.14^{* *}$ & $.17^{* * * *}$ & $.21 * * *$ & $.16^{* * *}$ & $.29 * * *$ & - & & & & & & \\
\hline DS7 & $.33 * * *$ & $.33 * * *$ & $.15^{* *}$ & $.20^{* * *}$ & $.25 * * *$ & $.13^{* *}$ & .08 & $.32 * * *$ & - & & & & & \\
\hline DS36 & $.16^{* * *}$ & $.37 * * *$ & .08 & $.13^{* *}$ & $.10^{*}$ & .03 & .08 & $.20 * * *$ & $.15^{* *}$ & - & & & & \\
\hline DS37 & $.17 * * *$ & $.21 * * *$ & $.16^{* * *}$ & $.17^{* * *}$ & .08 & $.12^{*}$ & $.14 * *$ & $.24 * * *$ & $.31 * * *$ & $.19 * * *$ & - & & & \\
\hline DS38 & $.19 * * *$ & $.12 *$ & .02 & .06 & .07 & .01 & .08 & .07 & $.22 * * *$ & .03 & $.17 * * *$ & - & & \\
\hline Veces Ira & $.42 * * *$ & $.19 * * *$ & $.20^{* * *}$ & $.23 * * *$ & .07 & .04 & $.17^{* * *}$ & $.15^{* *}$ & $.18 * * *$ & .07 & .07 & .04 & - & \\
\hline Ries. DL & $.47 * * *$ & $.57 * * *$ & $.28^{* * *}$ & $.29 * * *$ & $.28 * * *$ & .02 & $.23 * * *$ & $.24 * * *$ & $.25^{* * *}$ & $.19 * * *$ & $.15^{* *}$ & .06 & $.24 * * *$ & - \\
\hline Agr. DL & $.48 * * *$ & $.18 * * *$ & $.23^{* * *}$ & $.26 * * *$ & .06 & .03 & .04 & $.12 *$ & $.18 * * *$ & .08 & .07 & .02 & $.38 * * *$ & $.50^{* * *}$ \\
\hline \multirow{2}{*}{\multicolumn{15}{|c|}{ 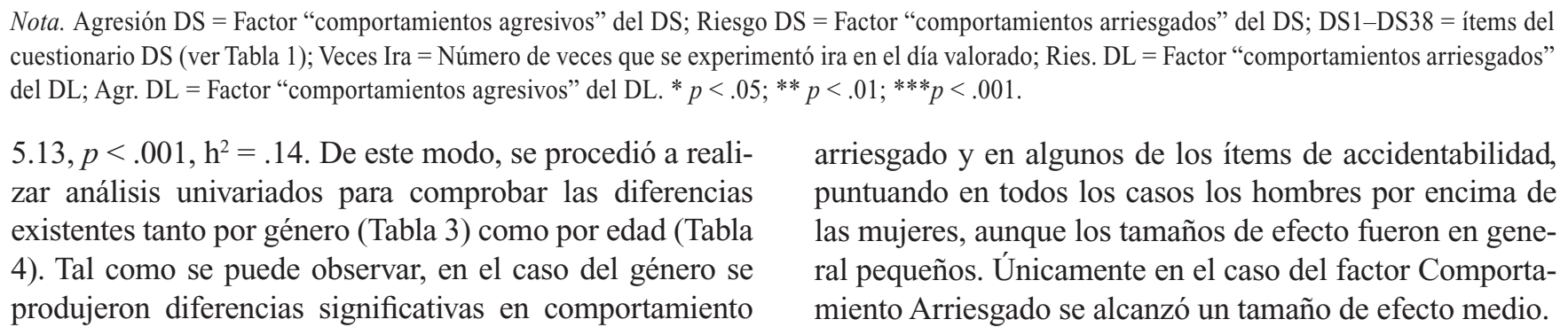 }} \\
\hline & & & & & & & & & & & & & & \\
\hline & & \multicolumn{4}{|c|}{ Hombres $(n=186)$} & \multicolumn{4}{|c|}{ Mujeres $(n=212)$} & \multicolumn{3}{|c|}{$F$} & \multicolumn{2}{|c|}{$\eta^{2}$} \\
\hline & & \multicolumn{2}{|l|}{$M$} & \multicolumn{2}{|c|}{$D T$} & \multicolumn{2}{|r|}{$M$} & \multicolumn{3}{|c|}{$D T$} & & & & \\
\hline \multicolumn{2}{|c|}{ Riesgo } & 9.02 & & \multicolumn{2}{|c|}{9.15} & \multicolumn{2}{|c|}{6.11} & \multicolumn{3}{|c|}{6.90} & $24.02 * * *$ & & \multicolumn{2}{|c|}{.06} \\
\hline \multicolumn{2}{|c|}{ Agresión } & 4.45 & & \multicolumn{2}{|c|}{5.92} & \multicolumn{2}{|c|}{4.23} & \multicolumn{3}{|c|}{5.70} & 2.06 & & \multicolumn{2}{|c|}{.01} \\
\hline \multicolumn{2}{|l|}{ DS1 } & 1.86 & & \multicolumn{2}{|c|}{1.20} & \multicolumn{2}{|c|}{2.03} & \multicolumn{3}{|c|}{1.21} & 0.90 & & \multicolumn{2}{|c|}{.00} \\
\hline \multicolumn{2}{|l|}{ DS2 } & 1.73 & & 1. & & & 1.48 & & 1.25 & & $6.68 * *$ & & .02 & \\
\hline DS3 & & 1.91 & & 1. & & & 1.62 & & 1.19 & & $9.75^{* *}$ & & .02 & \\
\hline DS4 & & 1.36 & & 1. & & & 1.71 & & 1.35 & & 3.51 & & .01 & \\
\hline DS5 & & 1.66 & & 1. & & & 1.97 & & 1.70 & & 3.42 & & .01 & \\
\hline DS6 & & 0.52 & & 1. & & & 0.32 & & 0.72 & & $8.19 * *$ & & .02 & \\
\hline DS7 & & 0.38 & & 0 . & & & 0.26 & & 0.67 & & $4.19^{*}$ & & .01 & \\
\hline DS36 & & 0.16 & & 0 . & & & 0.08 & & 0.30 & & 3.08 & & .01 & \\
\hline DS37 & & 0.28 & & 0 . & & & 0.30 & & 0.67 & & 0.26 & & .00 & \\
\hline DS38 & & 0.05 & & 0. & & & 0.05 & & 0.35 & & 0.20 & & .00 & \\
\hline
\end{tabular}

Nota . Riesgo = Factor "Comportamientos arriesgados"; Agresión = Factor "Comportamientos agresivos"; DS 1 - DS $38=$ ítems del cuestionario DS (ver Tabla 1). * $p<.05 ; * * p<.01 ; * * * p<.001$. 
En el caso de la edad, se apreciaron diferencias estadísticamente significativas en todas las variables excepto en los ítems relativos a la accidentabilidad Número de multas recibidas por conducir ilegalmente en el último año y Número de accidentes graves en el último año. En general, los conductores más jóvenes puntuaron por encima de los más mayores, con tamaños de efecto medios a grandes.

Tabla 4. Diferencias por edad en el Driving Surveya

\begin{tabular}{|c|c|c|c|c|c|c|c|c|c|c|}
\hline & \multicolumn{2}{|c|}{$<23(n=99)$} & \multicolumn{2}{|c|}{$23-30(n=62)$} & \multicolumn{2}{|c|}{$31-43(n=73)$} & \multicolumn{2}{|c|}{$>43(n=164)$} & \multirow[t]{2}{*}{$F$} & \multirow[t]{2}{*}{$\eta^{2}$} \\
\hline & $M$ & $D T$ & $M$ & $D T$ & $M$ & $D T$ & $M$ & $D T$ & & \\
\hline Riesgo & $10.27^{\mathrm{d}}$ & 8.12 & $10.44^{\mathrm{d}}$ & 9.28 & $8.66^{\mathrm{d}}$ & 8.62 & $4.13^{\mathrm{a}, \mathrm{b}, \mathrm{c}}$ & 6.09 & $21.72 *$ & .14 \\
\hline Agresión & $6.70^{\mathrm{c}, \mathrm{d}}$ & 7.22 & $6.60^{\mathrm{d}}$ & 6.29 & $4.45^{\mathrm{a}, \mathrm{d}}$ & 5.97 & $1.99^{\mathrm{a}, \mathrm{b}, \mathrm{c}}$ & 3.00 & $19.10^{*}$ & .13 \\
\hline DS 1 & $2.28^{\mathrm{d}}$ & 1.20 & $2.19^{\mathrm{d}}$ & 1.10 & $2.23^{\mathrm{d}}$ & 1.19 & $1.53^{\mathrm{a}, \mathrm{b}, \mathrm{c}}$ & 1.15 & $11.16^{*}$ & .08 \\
\hline DS 2 & $1.79^{\mathrm{d}}$ & 1.32 & $2.06^{\mathrm{d}}$ & 1.30 & $1.89^{\mathrm{d}}$ & 1.40 & $1.17^{\mathrm{a}, \mathrm{b}, \mathrm{c}}$ & 1.02 & $12.37 *$ & .09 \\
\hline DS 3 & $2.21^{\mathrm{d}}$ & 1.27 & 1.77 & 1.19 & $2.04^{\mathrm{d}}$ & 1.21 & $1.35^{\mathrm{a}, \mathrm{c}}$ & 1.09 & $14.95^{*}$ & .10 \\
\hline DS 4 & $2.16^{\mathrm{b}, \mathrm{c}, \mathrm{d}}$ & 1.48 & $1.50^{\mathrm{a}}$ & 1.57 & $1.59^{\mathrm{a}}$ & 1.22 & $1.17^{\mathrm{a}}$ & 1.05 & $10.65^{*}$ & .08 \\
\hline DS 5 & $1.99^{\mathrm{b}, \mathrm{d}}$ & 1.74 & $2.69^{\mathrm{a}, \mathrm{c}, \mathrm{d}}$ & 1.57 & $1.93^{\mathrm{b}}$ & 1.83 & $1.34^{\mathrm{a}, \mathrm{b}}$ & 1.56 & $10.20 *$ & .07 \\
\hline DS 6 & $0.72^{\mathrm{b}, \mathrm{d}}$ & 1.17 & $0.26^{\mathrm{a}}$ & 0.54 & 0.40 & 1.00 & $0.29^{\mathrm{a}}$ & 0.73 & $6.21 *$ & .05 \\
\hline DS 7 & $0.41^{\mathrm{d}}$ & 0.89 & $0.53^{\mathrm{d}}$ & 0.84 & $0.41^{\mathrm{d}}$ & 1.09 & $0.13^{\mathrm{a}, \mathrm{b}, \mathrm{c}}$ & 0.39 & $6.07 *$ & .05 \\
\hline DS 36 & 0.10 & 0.34 & 0.15 & 0.67 & 0.16 & 0.65 & 0.09 & 0.32 & 0.53 & .00 \\
\hline DS 37 & $0.40^{\mathrm{d}}$ & 0.65 & $0.45^{\mathrm{d}}$ & 0.80 & 0.34 & 0.79 & $0.13^{\mathrm{a}, \mathrm{b}}$ & 0.39 & $6.45^{*}$ & .05 \\
\hline DS 38 & 0.04 & 0.24 & 0.10 & 0.53 & 0.08 & 0.32 & 0.02 & 0.17 & 0.29 & .01 \\
\hline
\end{tabular}

Nota . Riesgo = Factor "Comportamentos arriesgados"; Agresión = Factor "Comportamientos agresivos"; DS 1 - DS 38 = ítems del cuestionario DS (ver Tabla 1). a Diferencias por edad según la prueba post-hoc GT2 de Hochberg. * $p<.001$.

\section{Predicción de la accidentabilidad en base a los comportamientos arriesgados y agresivos}

Finalmente se analizó la predicción de la accidentabilidad en base a los comportamientos arriesgados y agresivos al volante. Los resultados se detallan en la Tabla 5 (predicción de los eventos relacionados con la accidentabilidad en el último mes) y en la Tabla 6 (predicción de los eventos relacionados con la accidentabilidad en el último año). Tal como se puede observar, en el caso de los eventos ocurridos en el último mes los principales predictores fueron los comportamientos arriesgados y agresivos, que fueron significativos en los tres casos analizados. Por parte de los eventos sucedidos en el último año, nuevamente los comportamientos arriesgados y agresivos fueron los principales predictores, encontrándose menor número de relaciones significativas. Finalmente, los modelos de regresión propuestos explicaron mayor cantidad de varianza en el caso de los ítems referidos al último mes que en el caso de los ítems referidos al último año.

\section{Discusión}

La presente investigación tenía tres objetivos principales. En primer lugar, analizar las propiedades psico- métricas de la versión española del DS. En segundo lugar, analizar las diferencias por edad y por género en comportamiento arriesgado, en comportamiento agresivo, en eventos relacionados con la accidentabilidad y en la autopercepción del conductor en las dimensiones de calmado-colérico, poso agresivo- muy agresivo, cauteloso-temerario y seguro-inseguro. En tercer lugar, analizar el peso específico que tienen los comportamientos arriesgados y agresivos al volante con los eventos relacionados con la accidentabilidad.

De esta manera, este trabajo ha presentado la única adaptación española conocida del DS, y al igual que en la versión original (Deffenbacher et al., 2000), se halló que la estructura bifactorial propuesta (Factor $1=\mathrm{Com}$ portamientos arriesgados; Factor 2 = Comportamientos agresivos) ajustaba a los datos mejor que el modelo unifactorial. Además, ambos factores alcanzaron niveles satisfactorios de consistencia interna. En el caso del factor relativo a los comportamientos agresivos, un ítem fue suprimido por su baja correlación con el total del factor ("En el último mes, ¿cuántas veces rompiste o dañaste una parte del vehículo?"). Una posible razón de la baja relación de este ítem con el resto de su escala es que podría ser interpretado como un daño provocado por un accidente o como consecuencia de una colisión, estando 
Tabla 5. Regresión múltiple para predecir los eventos relacionados con la accidentabilidad en el último mes (todas las variables independientes se incluyeron simultáneamente en la ecuación)

\begin{tabular}{llllllllll}
\hline & $\mathrm{B}_{\mathrm{a}}$ & $\mathrm{ETB}_{\mathrm{a}}$ & $\beta_{\mathrm{a}}$ & $\mathrm{B}_{\mathrm{b}}$ & $\mathrm{ETB}_{\mathrm{b}}$ & $\beta_{\mathrm{b}}$ & $\mathrm{B}_{\mathrm{c}}$ & $\mathrm{ETB}_{\mathrm{c}}$ & $\mathrm{b}_{\mathrm{c}}$ \\
\hline Edad & -0.01 & 0.01 & -.05 & 0.00 & 0.00 & .02 & -0.01 & 0.00 & -.02 \\
Género $^{\mathrm{d}}$ & 0.43 & 0.17 & $.12^{*}$ & -0.15 & 0.09 & -.09 & -0.06 & 0.08 & -.04 \\
$\mathrm{CR}$ & 0.05 & 0.01 & $.23^{* *}$ & 0.02 & 0.01 & $.20^{* *}$ & 0.01 & 0.01 & $.15^{*}$ \\
$\mathrm{CA}$ & 0.03 & 0.02 & .08 & 0.04 & 0.01 & $.23^{* *}$ & 0.03 & 0.01 & $.23^{* *}$ \\
DS1 & 0.23 & 0.10 & $.16^{*}$ & -0.06 & 0.05 & -.08 & -0.07 & 0.05 & -.10 \\
DS2 & -0.02 & 0.10 & -.02 & -0.01 & 0.05 & -.01 & 0.03 & 0.04 & .04 \\
DS3 & 0.10 & 0.08 & .08 & 0.02 & 0.04 & .03 & 0.07 & 0.04 & $.11^{*}$ \\
DS4 & -0.02 & 0.07 & -.02 & 0.12 & 0.04 & $.17^{* *}$ & 0.06 & 0.03 & .09 \\
\hline
\end{tabular}

Nota. $\mathrm{X}_{\mathrm{a}}=$ Coeficientes relativos a la variable dependiente $=$ "En el último mes, ¿cuántas veces has perdido la concentración mientras conducías?" $\left(R^{2}=.19, p<.001\right) ; \mathrm{X}_{\mathrm{b}}=$ Coeficientes relativos a la variable dependiente = "En el último mes, ¿cuántas veces has tenido una pequeña pérdida del control del vehículo que estabas conduciendo?" $\left(R^{2}=.17, p<.001\right)$; $\mathrm{X}_{\mathrm{c}}=$ Coeficientes relativos a la variable dependiente $=$ "En el último mes, ¿cuántas veces has estado a punto de tener un accidente?" $\left(R^{2}=.16, p<.001\right)$. $\mathrm{B}=$ Coeficiente beta no-estandarizado; ETB = Error típico de beta; $\mathrm{b}=$ Beta estand; $\mathrm{CR}=$ Comportamientos arriesgados (DS); CA = Comportamientos agresivos (DS); DS1-DS4 = ítems del cuestionario DS (ver Tabla 1$) .{ }^{\mathrm{d}} 1=$ hombre; $2=$ mujer. ${ }^{*} p<.05 ; * * p<.001$.

Tabla 6. Regresión múltiple para predecir los eventos relacionados con la accidentabilidad en el último año

\begin{tabular}{ccccccccccc}
\hline & $\mathrm{B}_{\mathrm{a}}$ & $\mathrm{ETB}_{\mathrm{a}}$ & $\beta_{\mathrm{a}}$ & $\mathrm{B}_{\mathrm{b}}$ & $\mathrm{ETB}_{\mathrm{b}}$ & $\beta_{\mathrm{b}}$ & $\mathrm{B}_{\mathrm{c}}$ & $\mathrm{ETB}_{\mathrm{c}}$ & $\beta_{\mathrm{c}}$ \\
\hline Edad & 0.00 & 0.00 & .08 & -0.01 & 0.00 & $-.12^{*}$ & 0.00 & 0.00 & -.01 \\
Género $^{\mathrm{d}}$ & 0.01 & 0.05 & .01 & 0.01 & 0.07 & .01 & 0.01 & 0.03 & .02 \\
CR & 0.03 & 0.01 & $.45^{* *}$ & 0.01 & 0.01 & $.16^{*}$ & 0.00 & 0.00 & .01 \\
CA & -0.01 & 0.01 & -.07 & 0.00 & 0.01 & .00 & 0.01 & 0.00 & $.22^{* *}$ \\
DS1 & -0.01 & 0.03 & -.03 & 0.02 & 0.04 & .03 & -0.03 & 0.02 & -.12 \\
DS2 & 0.03 & 0.03 & .09 & 0.05 & 0.04 & .10 & 0.00 & 0.02 & .02 \\
DS3 & -0.03 & 0.02 & -.07 & -0.05 & 0.03 & -.11 & 0.01 & 0.02 & .05 \\
DS4 & -0.01 & 0.02 & -.02 & 0.04 & 0.03 & .08 & 0.00 & 0.01 & .01 \\
\hline
\end{tabular}

Nota. $X_{\mathrm{a}}=$ Coeficientes relativos a la variable dependiente: "En el último año, ¿cuántas veces te han multado por cometer una infracción al volante?" $\left(R^{2}=.15, p<.001\right) ; X_{\mathrm{b}}=$ Coeficientes relativos a la variable dependiente = "En el último año, ¿cuántas veces has tenido un accidente leve?" $\left(R^{2}=.08, p<.001\right) ; X=$ Coeficientes relativos a la variable dependiente = "En el último año, ¿cuántas veces has tenido un accidente grave?" $\left(R^{2}=.05, p=.019\right) . \mathrm{B}=$ Coeficiente beta no-estandarizado; ETB = Error típico de beta; $\beta=$ Beta estandarizada; CR $=$ Comportamientos arriesgados (DS); CA = Comportamientos agresivos (DS); DS1-DS4 = ítems del cuestionario DS (ver Tabla 1). ${ }^{\mathrm{d}} 1$

$=$ hombre; $2=$ mujer $* p<.05 ; * * p<.001$.

quizás más relacionado con comportamiento arriesgado o con un evento relacionado con la accidentabilidad que con el resultado de una respuesta agresiva.

Tal como se ha expuesto en la introducción, la razón para probar el ajuste tanto en un único factor como en dos radica en cuestiones teóricas. Mientras que los comportamientos arriesgados y agresivos pueden ser similares en cuento a la forma de expresión (el resultado conductual final), los mecanismos motivacionales que subyacen a cada uno de los dos es completamente diferente. En el caso de los comportamientos arriesgados, la principal motivación es la búsqueda de sensaciones, a la vez que en el caso de los comportamientos agresivos la principal motivación es causar un daño físico o psicológico a un tercero (Baron y Richardson, 1994; Berkowitz, 1993). Finalmente, los seis ítems que valoran la accidentabilidad fueron considerados como independientes, al igual que los cuatro ítems iniciales formulados como escalas visual-analógicas, debido a la baja consistencia interna que mostraron.

Con respecto a la validez convergente y divergente, los resultados mostraron correlaciones positivas y signi- 
ficativas de los factores Comportamiento arriesgado y Comportamiento agresivo del DS con los factores homólogos del DL, lo cual apoya la validez convergente de ambos factores. Además de esto, en el caso del factor relativo al comportamiento arriesgado, se observa una mayor correlación con el factor homólogo del DL que con el factor de comportamiento agresivo del DL y con el número de veces que se experimentó ira, evidenciando validez divergente. En el caso del factor de Comportamiento agresivo del DS, las correlaciones con los factores del DL y con el número de veces que se experimentó ira son muy similares, lo que podría implicar una baja validez divergente. Sin embargo, se aprecia una asociación más fuerte con las medidas de autopercepción de los continuos calmado-colérico y poco agresivo-muy agresivo que con la medida de cauteloso-temerario, lo cual apoya la validez divergente. En definitiva, el constructo de comportamiento arriesgado parece claro en cuanto a las diferencias que implica con el comportamiento agresivo. En cambio, el comportamiento agresivo parece tener aspectos en común con el riesgo. Estos aspectos en común entre ambos tipos de comportamiento han sido propuestos en estudios anteriores estudios (Dula y Geller, 2003), de modo que prácticamente todos los comportamientos agresivos implicarían un cierto grado de riesgo o peligro por cuanto la atención se ve refocalizada hacia el objeto de la agresión, pero los comportamientos arriesgados no necesariamente tendrían por qué implicar agresión (Herrero-Fernández, 2016).

En cuanto a las variables sociodemográficas analizadas, no se halló efecto significativo en la interacción género-edad en la frecuencia de conductas agresivas o arriesgadas. Analizando específicamente los efectos de la edad y el género, se comprobó que los hombres puntuaban por encima de las mujeres en el factor Comportamiento arriesgado, al igual que lo hacían por encima en las escalas visual - analógicas relativas a la autopercepción en los continuos "poco agresivo-muy agresivo" y "muy cauteloso-muy temerario" y en dos de los tres eventos relacionados con la accidentabilidad en el último mes. Con respecto al comportamiento arriesgado, la mayor parte de los estudios están de acuerdo en que los hombres muestran más frecuentemente este tipo de conducta (Rhodes y Pivik, 2011). Con respecto a la autopercepción como más agresivos y a la mayor tendencia a la accidentabilidad, se ha visto en algunos trabajos que los hombres tienden a cometer más infracciones y a sufrir accidentes más graves que las mujeres, lo cual a su vez se ha demostrado que está muy vinculado con la ira experimentada y expresada (Evans y Carrère, 1991; Reason, Manstead, Stradling, y Baxter, 1990). En cualquier caso, la relación del género con la ira y agresión al vo- lante no está clara, por cuanto existen estudios que avalan que los hombres son más proclives que las mujeres (Vanlaar, et al., 2008), mientras que otros proponen lo contrario (Sullman et al., 2014) y otros no encuentran diferencias (Herrero-Fernández y Fonseca-Baeza, 2017). En este sentido, el hecho de que no se hayan obtenido diferencias significativas en el factor Comportamiento agresivo, pero sí en la autopercepción en el continuo "poco agresivo - muy agresivo" podría interpretarse como que realmente no existen diferencias en la frecuencia de emisión de comportamientos específicos agresivos, pero sí en la idea autopercibida como aspecto cognitivo, quizás más relacionado con el autoconcepto. Sin embargo, este aspecto debe ser estudiado más profundamente en futuras investigaciones. Únicamente se ha encontrado un estudio al respecto que relacionaba ira autopercibida con agresión expresada, habiéndose obtenido una correlación baja entre ambas (Oliva, Calleja, y Hernández, 2012).

En cuanto a la edad, los resultados mostraron niveles más elevados en los más jóvenes que en los más mayores en los factores Comportamiento arriesgado y Comportamiento agresivo, al igual que en la autopercepción como más arriesgados, menos seguros y más agresivos. Estos datos son coherentes con otras investigaciones, que han puesto de relieve la mayor propensión a este tipo de conductas entre los conductores más jóvenes (Deffenbacher, Lynch, Oetting, y Swaim, 2002; Herrero-Fernández y Fonseca-Baeza, 2017).

Seguidamente se analizó la predicción de la accidentabilidad por parte de los comportamientos arriesgados y agresivos, así como de la autopercepción del conductor en las dimensiones establecidas. Los modelos de regresión mostraron resultados más robustos en base a la cantidad de varianza explicada y el número de relaciones significativas en el caso de los eventos relacionados con la accidentabilidad del último mes. Estos resultados sugirieron que, en el caso de los eventos del último mes, tanto el comportamiento arriesgado como el comportamiento agresivo se asocian de manera significativa a la accidentabilidad. Concretamente, la pérdida de concentración se asoció únicamente a comportamientos arriesgados, aunque en la literatura científica también hay evidencias de que los comportamientos agresivos podrían tener un efecto negativo sobre la atención, debido a la refocalización del foco de atención sobre el objeto de la ira, que habitualmente es el conductor del vehículo que indujo tal estado emocional (Deffenbacher et al., 2003). La diferencia de resultados en este caso con respecto a la presente investigación podría deberse a la forma de evaluar la distracción, ya que en el presente estudio se hacía por medio de un ítem que interrogaba acerca de la pérdida de con- 
centración. En cualquier caso, estudios han mostrado la relación entre distracciones y probabilidad de sufrir un accidente de tráfico (Batbold, 2017; Backaitis, 2006). Con respecto a la pérdida de control del vehículo, tanto los comportamientos arriesgados como los agresivos mostraron una relación significativa, lo cual es congruente con estudios previos (Deffenbacher, Lynch et al., 2003; Deffenbacher, Lynch, Oetting, y Yingling, 2001). Finalmente, con respecto al hecho de haber estado a punto de sufrir un accidente de tráfico, los comportamientos agresivos mostraron una relación más fuerte que los comportamientos arriesgados. En este sentido, hay estudios que han mostrado que la accidentabilidad está asociada con ambos tipos de comportamiento, aunque diseños más sofisticados han propuesto que podría tratarse de una relación de mediación, de manera que los comportamientos arriesgados mediarían en la relación entre conducta agresiva y accidentabilidad (Herrero-Fernández y Fonseca-Baeza, 2017; Zhang, Chan, Xue, Zhang, y Tao, 2019).

En cuanto a los eventos del último año, los comportamientos arriesgados parecen tener una mayor repercusión. Teniendo en cuenta que se un análisis retrospectivo mucho más extenso (un año, frente a un mes del caso anterior), estos resultados menos robustos que en el anterior caso podrían deberse a un sesgo de memoria en cuanto a la recuperación de información. En cualquier caso, estos resultados se corresponden con otras investigaciones, que han concluido que ambos tipos de comportamiento, aunque especialmente los arriesgados, se asocian con la accidentabilidad (Chraif, Anitei, Burtaverde, y Mihaila, 2016; Qu, et al., 2015; Zhang y Chan, 2016).

Finalmente, la edad y el género apenas tuvieron relación significativa con la accidentabilidad en ninguno de los dos periodos de tiempo valorados. En el caso del género, únicamente se asoció significativamente con la pérdida de concentración, lo cual es coherente con estudios anteriores que se sugieren que las mujeres tienden a distraerse más que los hombres (Bone y Mowen, 2006; Schwebel et al., 2006). Por su parte, la edad se asoció negativamente con el hecho de haber sufrido un accidente leve.

\section{Limitaciones del estudio y conclusiones}

Con todo esto, el presente estudio cuenta con dos limitaciones. En primer lugar, la muestra estudiada fue seleccionada incidentalmente de dos regiones de España, lo cual podría limitar la generalización de los resultados. En este sentido, se recomienda confirmar los resultados obtenidos en futuras investigaciones tomando participantes de distintas regiones, tratando de contar con muestras realmente representativas. En segundo lu- gar, la presente investigación se ha realizado sobre variables medidas con auto-informes. Si bien hay estudios que muestran la validez de este procedimiento (Lajunen y Summala, 2003), futuras investigaciones deberían verificar los resultados obtenidos por medio de otro tipo de metodologías más objetivas y con mayor validez ecológica, como tareas de simulación.

En conclusión, el DS ha evidenciado unas propiedades psicométricas adecuadas para poder ser utilizado en la evaluación de la autopercepción de los conductores en los términos indicados, en la frecuencia de comportamientos arriesgados y agresivos al volante y en la accidentabilidad. Además, se ha arrojado luz sobre la relación entre riesgo, agresión y accidentabilidad, lo cual puede ser útil de cara a diseñar programas terapéuticos que busquen la modificación de comportamientos en conductores proclives a generar accidentes de tráfico e infractores. Sin embargo, los resultados obtenidos en este estudio deberían ser confirmados en futuras investigaciones y con muestras específicas de conductores infractores.

\section{Conflictos de intereses}

Los autores declaran que no existen conflictos de intereses.

\section{Referencias}

Backaitis, S. (2006). Driver's inattention and distraction in the causation of traffic accidents. Transport Means 2006, Proceedings (pp. 25-28).

Baron, R. A., \& Richardson, D. R. (1994). Human aggression (2 ed.). New York: Plenum Press.

Batbold, N. (2017). Driver's attention and anxiety: Relation with traffic accidents. Journal of the Neurological Sciences, 381, 282-282.

Berkowitz, L. (1993). Aggression and its causes, consequences and control. New York: McGraw-Hill.

Bollen, K. A. (1989). Structural equations with latent variables. New York: Wiley.

Bone, S. A., \& Mowen, J. C. (2006). Identifying the traits of aggressive and distracted drivers: A hierarchical trait model approach. Journal of Consumer Behaviour, 5(5), 454-464.

Brewer, A. M. (2000). Road rage: What, who, when, where and how?. Transport Review, 20(1), 49-64.

Cohen, J. (1988). Statistical power analysis for the behavioral sciences (2nd ed.). Hillsdale, NJ: Erlbaum.

Chraif, M., Anitei, M., Burtaverde, V., \& Mihaila, T. (2016). The link between personality, aggressive driving, and risky driving outcomes: Testing a theoretical model. Journal of Risk Research, 19(6), 780-797.

Deffenbacher, J. L., Deffenbacher, D. M., Lynch, R. S., \& Richards, T. L. (2003). Anger, aggression and risky behavior: A comparison of high and low anger drivers. Behaviour Research and Therapy, 41(6), 701-718. 
Deffenbacher, J. L., Filetti, L. B., Richards, T. L., Lynch, R. S., \& Oetting, E. R. (2003). Characteristics of two groups of angry drivers. Journal of Counseling Psychology, 50(2), 123-132.

Deffenbacher, J. L., Huff, M. E., Lynch, R. S., Oetting, E. R., \& Salvatore, N. F. (2000). Characteristics and treatment of highanger drivers. Journal of Counseling Psychology, 47(1), 5-17.

Deffenbacher, J. L., Lynch, R. S., Filetti, L. B., Dahlen, E. R., \& Oetting, E. R. (2003). Anger, aggression, risky behavior, and crash-related outcomes in three groups of drivers. Behaviour Research and Therapy, 41(3), 333-349.

Deffenbacher, J. L., Lynch, R. S., Oetting, E. R., \& Swaim, R. C. (2002). The Driving Anger Expression Inventory: A measure of how people express their anger on the road. Behaviour Research and Therapy, 40(6), 717-737.

Deffenbacher, J. L., Lynch, R. S., Oetting, E. R., \& Yingling, D. A. (2001). Driving anger: Correlates and a test of state-trait theory. Personality and Individual Differences, 31(8), 1321-1331.

DGT. (2017). Anuario Estadístico de Accidentes - 2017. Recuperado de: http://www.dgt.es/Galerias/seguridad-vial/ estadisticas-e-indicadores/publicaciones/anuario-estadisticode-accidentes/Anuario-estadistico-de-accidentes-2017.pdf.

Dula, C. S., \& Geller, E. S. (2003). Risky, aggressive, or emotional driving: Addressing the need for consistent communication in research. Journal of Safety Research, 34(5), 559-566.

Elander, J., West, R., \& French, D. (1993). Behavioral correlates of individual differences in road-traffic crash risk: An examination of methods and findings. Psychological Bulletin, 113(2), 279-294.

Evans, G. W., \& Carrère, S. (1991). Traffic congestion, perceived control, and psychophysiological stress among urban bus drivers. Journal of Applied Psychology, 76(5), 658-663.

Hambleton, R. K. (1996). Adaptación de tests para su uso en diferentes idiomas y culturas: Fuentes de error, posibles soluciones y directrices prácticas. En J. Muñiz (Ed.), Psicometría (pp. 207-238). Madrid: Universitas.

Herrero-Fernández, D. (2011a). Adaptación psicométrica de la versión reducida del Driving Anger Scale en una muestra española. Diferencias por edad y sexo. Anales de Psicología, 27(2), 544-549.

Herrero-Fernández, D. (2011b). Psychometric adaptation of the Driving Anger Expression Inventory in a Spanish sample. Differences by age and gender. Transportation Research Part F: Traffic Psychology and Behaviour, 14, 324-329.

Herrero-Fernández, D. (2016). Psychophysiological, subjective and behavioral differences between high and low anger drivers in a simulation task. Transportation Research Part F: Traffic Psychology and Behavior, 42, 365-375.

Herrero-Fernández, D. (2017). Procedimientos psicológicos a tener en cuenta en las inspecciones de seguridad vial. Carreteras, 211, 17-24.

Herrero-Fernández, D., \& Fonseca-Baeza, S. (2017). Angry thoughts in Spanish drivers and their relationship with crashrelated events. The mediation effects of aggressive and risky driving. Accident Analysis and Prevention, 106, 99-108.
Herrero-Fernández, D., Fonseca-Baeza, S., y Pla-Sancho, S. (2014). Estructura factorial del Driving Log (DL) en una muestra española [Factorial structure of the Driving Log (DL) in a Spanish sample]. Revista de Psicología, 32(1), 67-90.

Iliescu, D., \& Sârbescu, P. (2013). The relationship of dangerous driving with traffic offenses: A study on an adapted measure of dangerous driving. Accident Analysis and Prevention, 51, 33-41.

Lajunen, T., \& Summala, H. (2003). Can we trust self-reports of driving? Effects of impression management on driver behaviour questionnaire responses. Transportation Research Part F: Traffic Psychology and Behaviour, 6(2), 97-107.

Montoro, L., Useche, S., Alonso, F., \& Cendales, B. (2018). Work Environment, Stress, and Driving Anger: A Structural Equation Model for Predicting Traffic Sanctions of Public Transport Drivers. International Journal of Environmental Research and Public Health, 15(3), 497-508.

Oliva, F., Calleja, N., \& Hernández, R. (2012). Scale of beliefs about anger in combat sports among Mexican Athletes. Revista Internacional de Medicina y Ciencias de la Actividad Fisica y del Deporte, 12(45), 110-121.

Qu, W., Ge, Y., Xiong, Y., Carciofo, R., Zhao, W., \& Zhang, K. (2015). The relationship between mind wandering and dangerous driving behavior among Chinese drivers. Safety Science, 78, 41-48.

Reason, J. T., Manstead, A., Stradling, S., \& Baxter, J. S. (1990). Errors and violations on the roads: A real distinction? Ergonomics, 33(10), 1315-1332.

Rhodes, N., \& Pivik, K. (2011). Age and gender differences in risky driving: The roles of positive affect and risk perception. Accident Analysis and Prevention, 43(3), 923-931.

Schwebel, D. C., Ball, K. K., Severson, J., Barton, B. K., Rizzo, M., \& Viamonte, S. M. (2007). Individual difference factors in risky driving among older adults. Journal of Safety Research, 38(5), 501-509.

Schwebel, D. C., Severson, J., Ball, K. K., \& Rizzo, M. (2006). Individual difference factors in risky driving: The roles of anger/hostility, conscientiousness, and sensation-seeking. Accident Analysis y Prevention, 38(4), 801-810.

Smith, A. P. (2016). A UK survey of driving behaviour, fatigue, risk taking and road traffic accidents. BMJ Open, 6(8).

Sullman, M. J. M., Stephens, A. N., \& Yong, M. (2014). Driving anger in Malaysia. Accident Analysis and Prevention, 71, 1-9.

Vanlaar, W., Simpson, H., Mayhew, D., \& Robertson, R. (2008). Aggressive driving: A survey of attitudes, opinions and behaviors. Journal of Safety Research, 39(4), 375-381.

Zhang, T., \& Chan, A. H. S. (2016). The association between driving anger and driving outcomes: A meta-analysis of evidence from the past twenty years. Accident Analysis and Prevention, 90, 50-62.

Zhang, T. R., Chan, A. H. S., Xue, H. J., Zhang, X. Y., \& Tao, D. (2019). Driving anger, aberrant driving behaviors, and road crash risk: Testing of a mediated model. International Journal of Environmental Research and Public Health, 16(3), 297-309. 